\title{
Correction to: Gingerol inhibits cisplatin-induced acute and delayed emesis in rats and minks by regulating the central and peripheral $5-\mathrm{HT}$, SP, and DA systems
}

\author{
Li Tian ${ }^{1} \cdot$ Weibin Qiann ${ }^{2,3} \cdot$ Qiuhai Qian ${ }^{4} \cdot$ Wei Zhang $^{3}$ (I) $\cdot$ Xinrui Cai $^{5}$
}

Published online: 17 January 2020

(c) The Author(s) 2020

\section{Correction to: Journal of Natural Medicines https://doi.org/10.1007/s11418-019-01372-x}

In the original publication of the article, Figures 2, 3, 5, 11 and 13 were published incorrectly. The correct version of Fig. 2, 3, 5, 11 and 13 are given below,

Open Access This article is licensed under a Creative Commons Attribution 4.0 International License, which permits use, sharing, adaptation, distribution and reproduction in any medium or format, as long as you give appropriate credit to the original author(s) and the source, provide a link to the Creative Commons licence, and indicate if changes were made. The images or other third party material in this article are included in the article's Creative Commons licence, unless indicated otherwise in a credit line to the material. If material is not included in

The original article can be found online at https://doi.org/10.1007/ s11418-019-01372-x.

Wei Zhang

doctorzwjn@126.com

$\triangle$ Xinrui Cai

doctorcai@163.com

1 First Clinical Medical College, Shandong University of Traditional Chinese Medicine, Jinan, Shandong, People's Republic of China

2 Postdoctoral Mobile Station, Shandong University of Traditional Chinese Medicine, Jinan, Shandong, People's Republic of China

3 Department of Lung Disease, Affiliated Hospital of Shandong University of Traditional Chinese Medicine, No. 16369 Jingshi Road, Lixia District, Jinan, Shandong, People's Republic of China

4 Department of Endocrinology, Affiliated Hospital of Shandong University of Traditional Chinese Medicine, Jinan, Shandong, People's Republic of China

5 Department of Traditional Chinese Medicine, Shandong Academy of Occupational Health and Occupational Medicine, Shandong First Medical University and Shandong Academy of Medical Sciences, No. 17 Yuxing Road, Central District, Jinan, Shandong, People's Republic of China the article's Creative Commons licence and your intended use is not permitted by statutory regulation or exceeds the permitted use, you will need to obtain permission directly from the copyright holder. To view a copy of this licence, visit http://creativecommons.org/licenses/by/4.0/.

Publisher's Note Springer Nature remains neutral with regard to jurisdictional claims in published maps and institutional affiliations. 


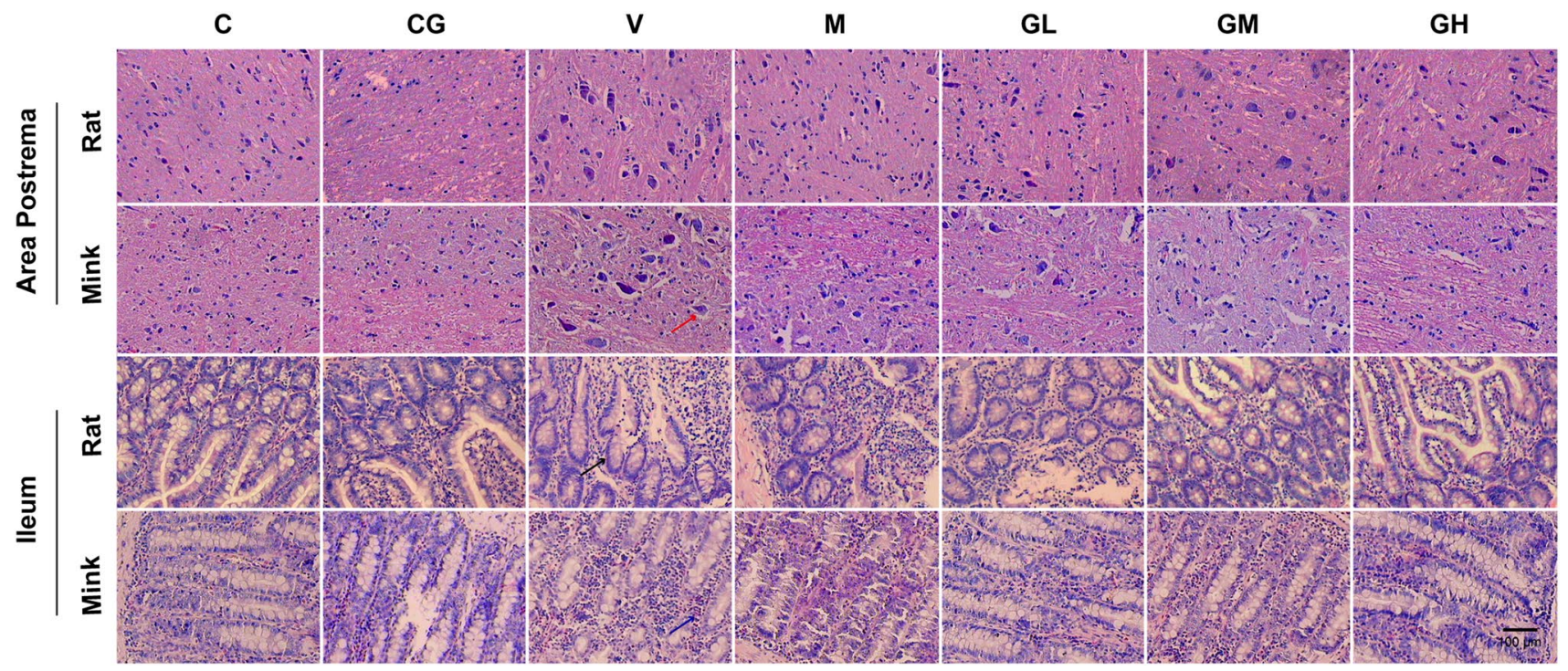

Fig. 2 Representative $H \& E$ staining in area postrema along with ileum of rats and minks ( $100 \times$ magnification). The figures show the H\&E staining in area postrema along with ileum of rats and minks (rats: $n=5$, minks: $n=6$ ). Bar indicates $100 \mu \mathrm{m}$. $C$ normal control group, $C G$ simple gingerol control group, $V$ cisplatin control group,
$M$ cisplatin + metoclopramide group, $G L$ cisplatin + low-dose gingerol group, $G M$ cisplatin + middle-dose gingerol group, $G H$ cisplatin + high-dose gingerol group. The red arrow shows the nerve cell, black arrow shows the epithelial cell, and blue arrow shows the inflammatory cell 


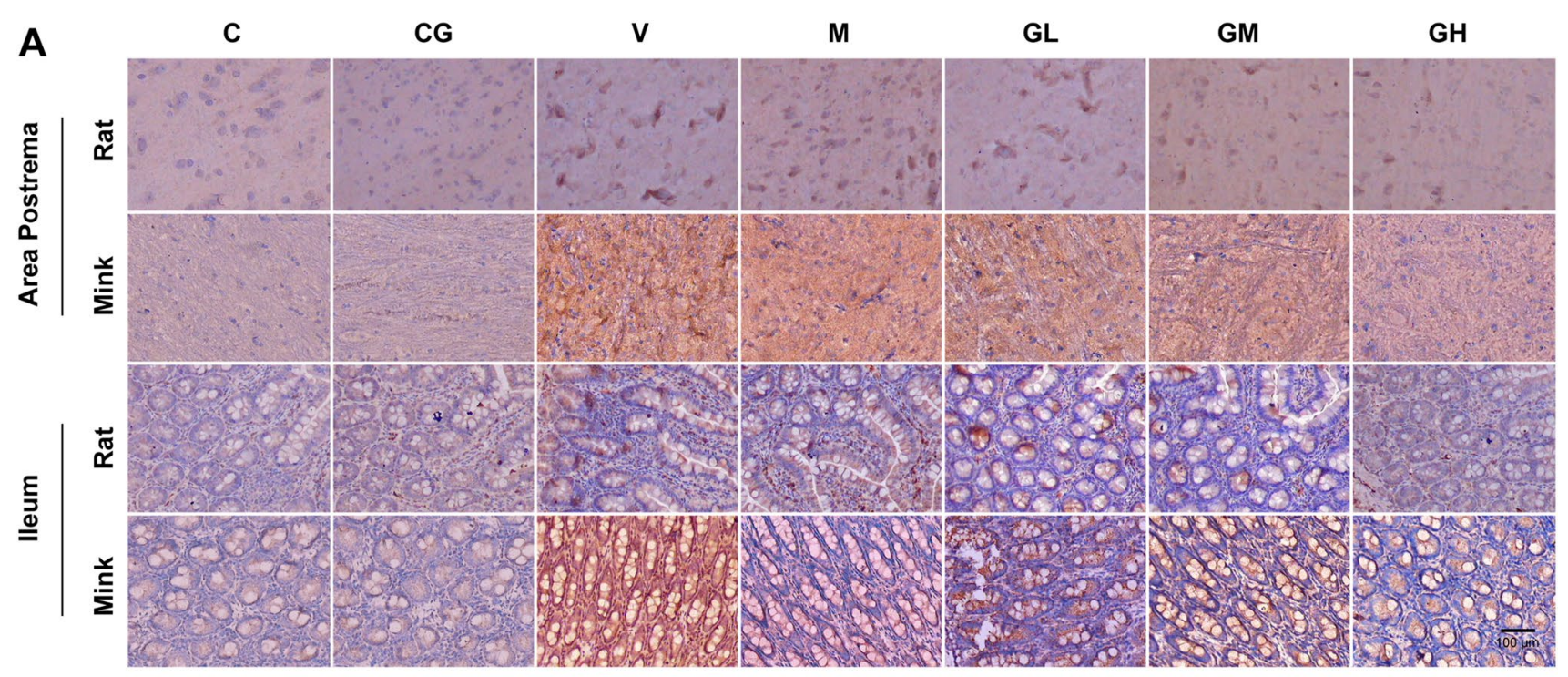

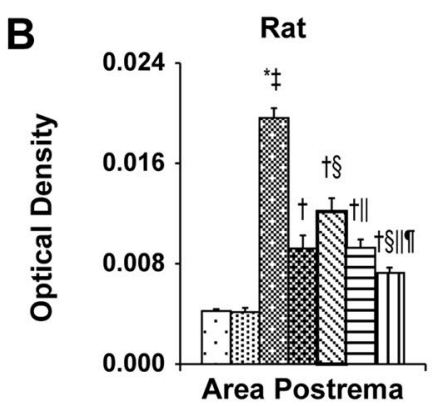
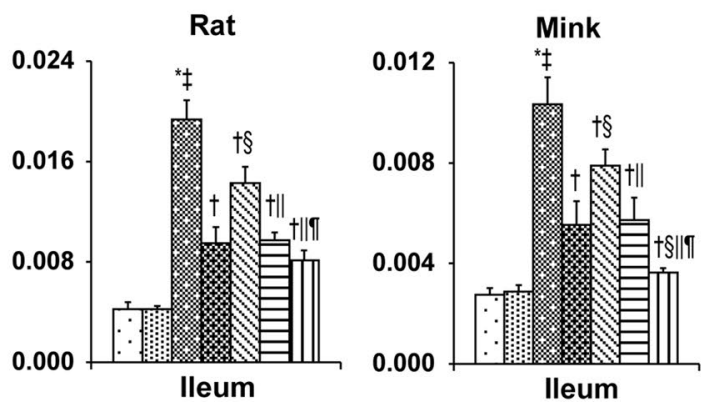

$\square \mathrm{C}$

国 CG

国

ำ $M$

$\otimes G L$

曰GM
Fig. 3 5-HT immunostaining expression in area postrema in addition to ileum of rats and minks. a Immunohistochemistry manifestation of 5-HT in area postrema in addition to ileum of rats and minks (rats: $n=5$, minks: $n=6$ ). Bar indicates $100 \mu \mathrm{m}$. b Mean optical density values of $5-\mathrm{HT}$. The images were quantified by Image-Pro Plus. $C$ normal control group, $C G$ simple gingerol control group, $V$ cisplatin control group, $M$ cisplatin + metoclopramide group, $G L$ cisplatin + low-dose gingerol group, $G M$ cisplatin + middle-dose gingerol group, $G H$ cisplatin + high-dose gingerol group. ${ }^{*} P<0.05$ vs. Group $\mathrm{C},{ }^{\ddagger} P<0.05$ vs. Group CG, ${ }^{\dagger} P<0.05$ vs. Group V, ${ }^{\S} P<0.05$ vs. Group M, "P<0.05 vs. Group GL, ${ }^{\pi} P<0.05$ vs. Group GM. $5-H T$ 5-tyrosine hydroxylase 


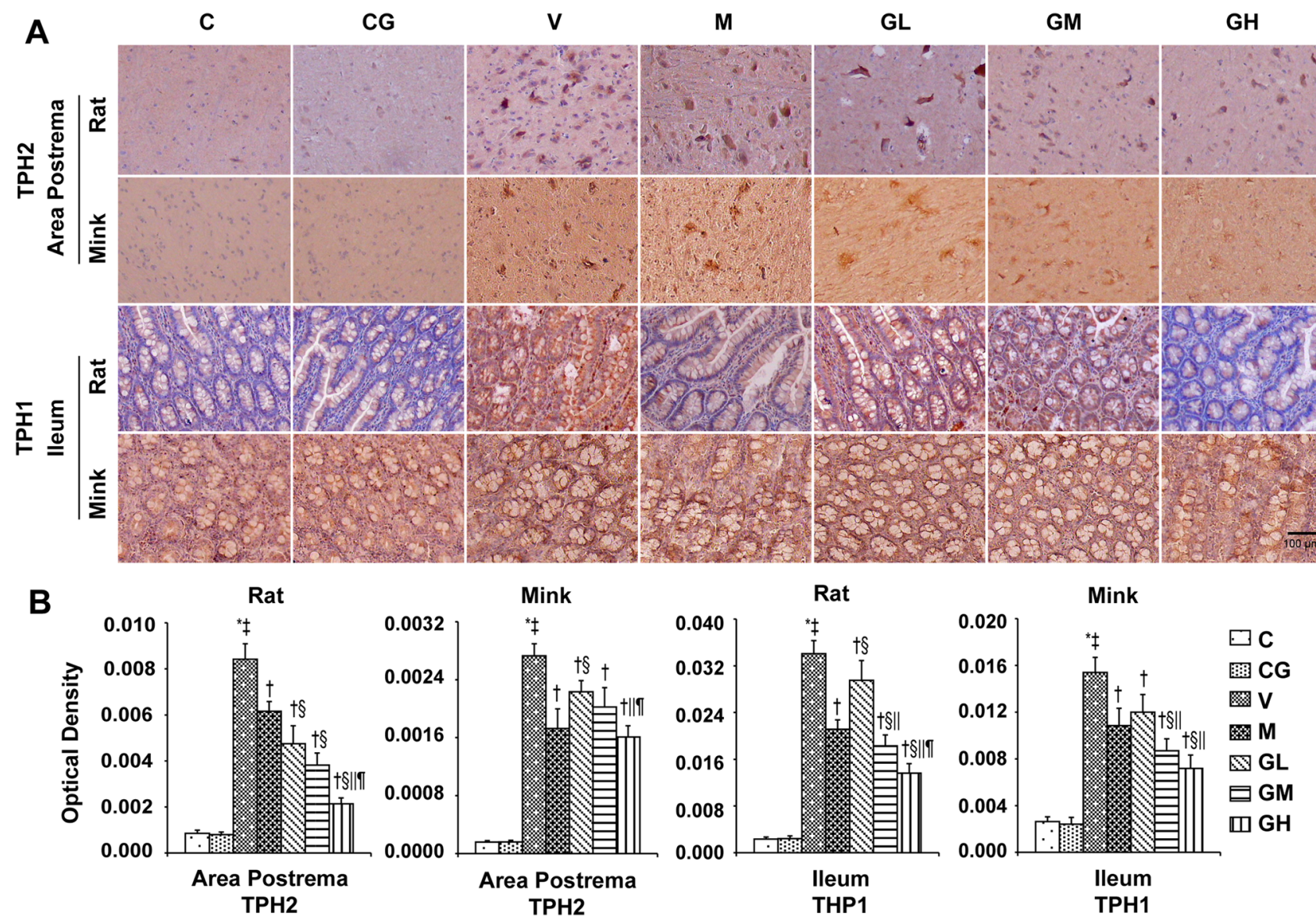

Fig. $5 \mathrm{TPH}$ immunostaining manifestation in area postrema as well as ileum of rats and minks. a Immunohistochemistry manifestation of $\mathrm{TPH}_{2}$ in area postrema of rats plus minks, and $\mathrm{TPH}_{1}$ in ileum of rats and minks (rats: $n=5$, minks: $n=6$ ). Bar indicates $100 \mu \mathrm{m}$. b Mean optical density values of $\mathrm{TPH}_{2}$ and $\mathrm{TPH}_{1}$. The images were quantified by Image-Pro Plus. $C$ normal control group, $C G$ simple gingerol control group, $V$ cisplatin control group, $M$ cisplatin + meto-

clopramide group, $G L$ cisplatin + low-dose gingerol group, $G M$ cisplatin + middle-dose gingerol group, $G H$ cisplatin + high-dose gingerol group. ${ }^{*} P<0.05$ vs. Group $C,{ }^{\ddagger} P<0.05$ vs. Group $C G,{ }^{\dagger} P<0.05$ vs. Group $\mathrm{V},{ }^{\S} P<0.05$ vs. Group M, $P<0.05$ vs. Group GL, ${ }^{\mathbb{T}} P<0.05$ vs. Group GM. $T P H_{1}$ tryptophan hydroxylase $1, T P H_{2}$ tryptophan hydroxylase 2 


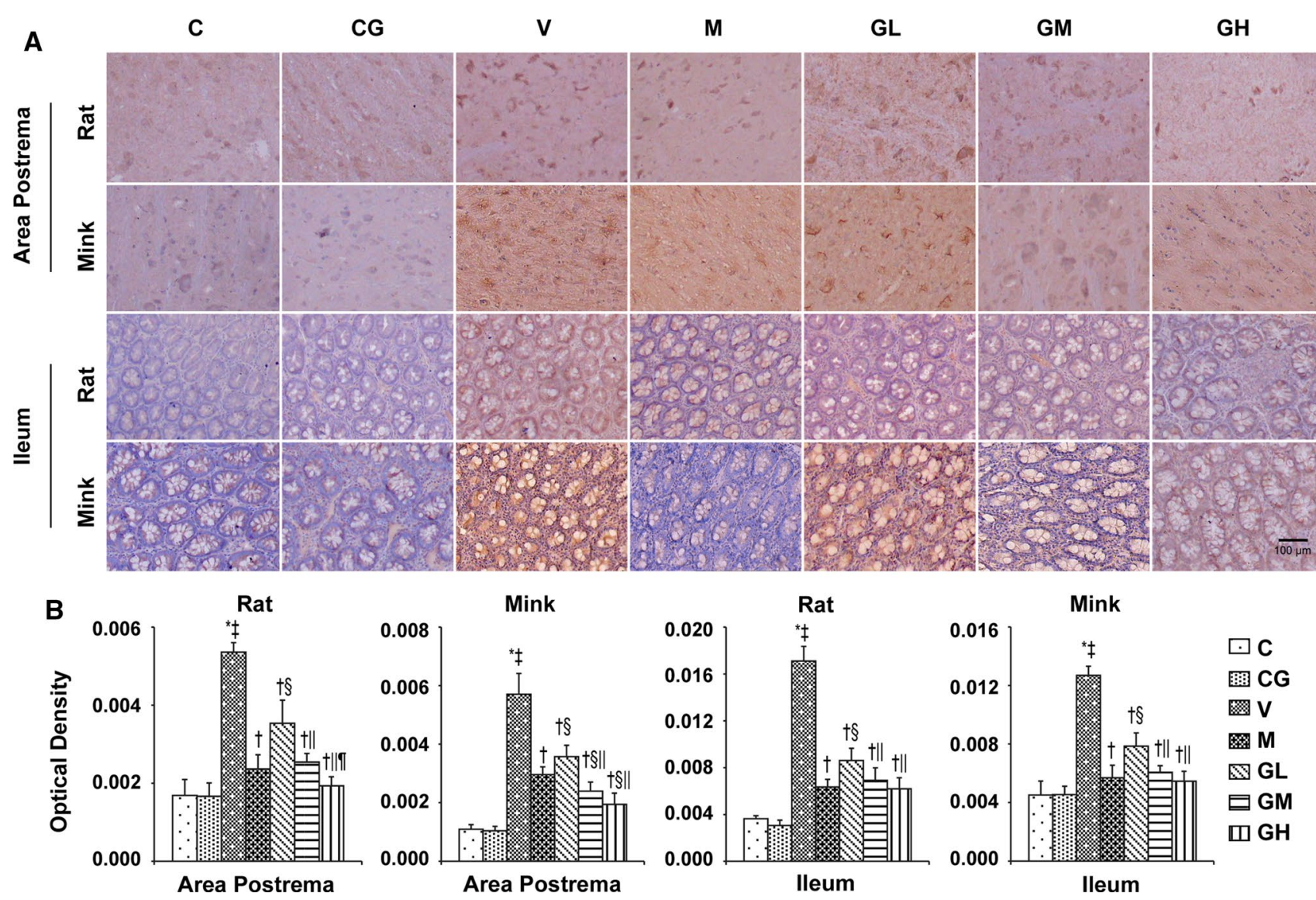

Fig. 11 DA immunostaining expression in area postrema in addition to ileum of rats and minks. a Immunohistochemistry manifestation of DA in area postrema plus ileum of rats and minks (rats: $n=5$, minks: $n=6$ ). Bar indicates $100 \mu \mathrm{m}$. b Mean optical density values of DA. The images were quantified by Image-Pro Plus. $C$ normal control group, $C G$ simple gingerol control group, $V$ cisplatin control group,

$M$ cisplatin + metoclopramide group, $G L$ cisplatin + low-dose gingerol group, $G M$ cisplatin + middle-dose gingerol group, $G H$ cisplatin + high-dose gingerol group. ${ }^{*} P<0.05$ vs. Group $\mathrm{C}, \stackrel{\stackrel{\varpi}{\ddagger}}{P}<0.05$ vs. Group CG, ${ }^{\dagger} P<0.05$ vs. Group V, ${ }^{\S} P<0.05$ vs. Group $\mathrm{M},{ }^{\|} P<0.05$ vs. Group GL, ${ }^{\pi} P<0.05$ vs. Group GM. $D A$ dopamine 


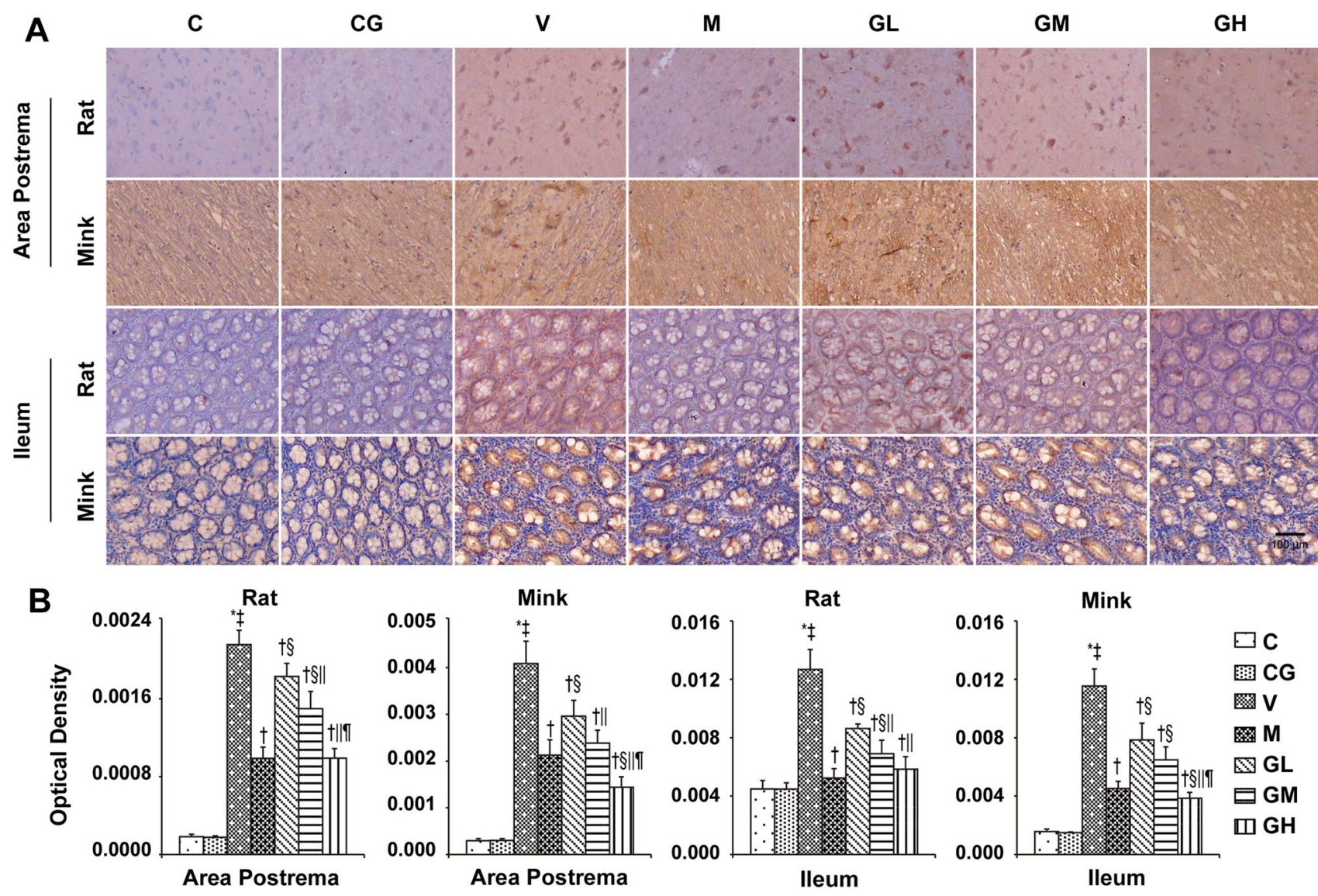

Fig. $13 \mathrm{TH}$ immunostaining manifestation in area postrema in addition to ileum of rats and minks. a Immunohistochemistry manifestation of TH in area postrema plus ileum of rats and minks (rats: $n=5$, minks: $n=6$ ). Bar indicates $100 \mu \mathrm{m}$. b Mean optical density values of $\mathrm{TH}$. The images were quantified by Image-Pro Plus. $C$ normal control group, $C G$ simple gingerol control group, $V$ cisplatin control group,

$M$ cisplatin + metoclopramide group, $G L$ cisplatin + low-dose gingerol group, $G M$ cisplatin + middle-dose gingerol group, $G H$ cisplatin + high-dose gingerol group. ${ }^{*} P<0.05$ vs. Group $\mathrm{C},{ }^{\ddagger} P<0.05$ vs. Group CG, ${ }^{\dagger} P<0.05$ vs. Group V, ${ }^{\S} P<0.05$ vs. Group M, $" P<0.05$ vs. Group GL, ${ }^{\mathbb{T}} P<0.05$ vs. Group GM. $T H$ tyrosine hydroxylase 